\title{
The Relationship between Psychological Suzhi and Mental Health among Chinese College Students
}

\author{
Xinqiang Wang1*, Xueqi Zhang1, Dajun Zhang2 \\ ${ }^{1}$ School of Psychology \& Center of Mental Health Education and Research, Jiangxi Normal University, Nanchang, \\ China \\ ${ }^{2}$ Center of Mental Health Education and Research, Southwest University, Chongqing, China \\ Email: *xinqiangw101@163.com
}

Received 28 March 2016; accepted 7 May 2016; published 10 May 2016

Copyright (C) 2016 by authors and Scientific Research Publishing Inc.

This work is licensed under the Creative Commons Attribution International License (CC BY). http://creativecommons.org/licenses/by/4.0/

c) (i) Open Access

\begin{abstract}
Object: To explore the relationship between psychological suzhi and mental health among Chinese college students, and to gain psychological suzhi factors that are predictors for mental health. Method: By using stratified sampling method, an investigation was conducted among 734 subjects. They were assessed with the College Student Psychological Suzhi Scale (CSPS, including 3 subscales, 28 factors) and General Health Questionnaire-20 item (GHQ-20, including 3 subscales). Results: 1) Psychological suzhi score for Chinese college students had negative correlation with the score of GHQ-20, GHQ-depression and GHQ-anxiety $(p<0.001)$, and positive correlation with the score of GHQ-self-affirmation $(p<0.001) ; 2)$ Psychological suzhi score for Chinese college students was predictor of the score for GHQ-20 and its subscales namely GHQ-self-affirmation, GHQ-depression, and GHQ-anxiety $(\beta=-0.448,0.439,-0.262,-0.259, p<0.001)$; the variance explained by the score of GHQ-20 and its subscales were $19.9 \%, 19.1 \%, 6.7 \%, 6.5 \%$; 3 ) There were 12 psychological suzhi factors that were predictors for GHQ-self-affirmation which was known as the positive indicator of mental health $(p<0.05) ; 11$ psychological suzhi factors were predictors for GHQ-depression and GHQ-anxiety which was known as the negative indicator of mental health ( $p<$ 0.05). Conclusion: There exists a correlation between psychological suzhi and mental health, particularly in positive mental health. Indeed, the psychological suzhi factors are able to enhance the pertinence of mental health education.
\end{abstract}

\section{Keywords}

Chinese College Students, Mental Health, Psychological Suzhi, Dual-Factor Model of

"Corresponding author.

How to cite this paper: Wang, X.Q., Zhang, X.Q. and Zhang, D.J. (2016) The Relationship between Psychological Suzhi and Mental Health among Chinese College Students. Journal of Biosciences and Medicines, 4, 21-29.

http://dx.doi.org/10.4236/jbm.2016.45002 


\section{Mental Health}

\section{Introduction}

As a relatively higher educational group, college students are the main impetus to the development of countries in the future. Therefore, the development of contemporary college students is always concerned by a community. In the several factors which can influence the development of college students, the requirement for finishing school smoothly and adapting to society is mental health [1]. But under the exam-oriented education, middle schools tend to ignore the cultivation of students' inside mental feeling and psychological suzhi [2]. Along with the increasingly drastic social competition (external stress events faced by individuals was increased), and the increasingly divorce rate (social support system owned by individuals was broken or reduced), students can't adjust their mental situation more and more. This negative result carries on to college; the level of college freshmen's mental health was relatively steady from 1986 to 2010; despite the government's spending on the students' mental health education more and more, however their mental health situation did not get positive [3]. To sum up, discussing how to improve mental health level of college students effectively is imminent.

Chinese scholars put forward the concept of psychological suzhi for how to improve mental health status of college students. Psychological suzhi is based on the physiological condition, internalizes the obtained stimuli from external world as psychological quality which is stable, basic, and implicit, has basic, derived and development capabilities, and keeps in close contact with social adaptive behavior and creative behavior. Psychological suzhi is a mental quality system, which consists of three dimensions namely cognition quality, personality quality, and adaptability (adaptive capacity). There are crucial differences between psychological suzhi and mental health: psychological suzhi is a kind of "quality”, and mental health is a kind of "state”. Psychological suzhi is an endogenous element of mental health, which can be seen as psychological fitness (constitution) of individual fitness (constitution) [4]-[6]. Cognition quality refers to individual characteristics which express in the cognitive process. Personality quality refers to personality characteristics which express in everyday life. Cognition quality and personality quality are all content elements of psychological suzhi. Adaptability (adaptive capacity) is theoretically an individual on the basis of cognitive quality and personality quality through interaction within a specific situation which includes the selection, adaptation and change in the environment. It is the habitual behaviour when an individual is able to get along well with the surroundings. It belongs to the function elements of psychological suzhi [6]. The relationship between psychological suzhi and mental health was discussed by many researchers since the concept of psychological suzhi has been put forward. The theoretical research of the relationship showed that "psychological suzhi is a trait, but mental health is a state, the state is unable to change itself, the only way to change state is to change quality [7]", and also emphasized that "psychological suzhi is endogenous, mental health is reactive”. Therefore, the fundamental way to improve mental health condition is to enhance psychological suzhi [5] [8] [9]. Emerging research suggests that individuals who have higher psychological suzhi suffer from less stress, and they can recover by themselves when they face stress. Instead, individuals who have lower psychological suzhi suffer from more stress, and they cannot recover by themselves easily [6]. In the relevant empirical research, these points have been confirmed. Wang and Zhang suggested that middle school students with higher psychological suzhi had a higher level of mental health, and psychological suzhi is a protective factor in the process that external events influence individual mental health [10]. Other studies also provide support for the significant association between psychological suzhi and mental health, such as there existed a significant correlation between psychological suzhi and mental health among army's young soldiers [11]. However, there is a lack of related empirical research for Chinese college students.

In conclusion, this study proposes to have a systematic empirical research for the relationship between psychological suzhi and mental health among College students, based on the theory of the relationship [6] [9]. In the previous research, College students' mental health was mostly measured by negative mental health scale, such as SCL-90 [12]. However previous studies on the connotative structure of mental health showed that the mental health includes positive and negative two important aspects, not only is the absence of mental illness, or the high-level subject well-being, but also is a complete state by combining the two, namely compete mental health [13]-[15]. From this perspective, the present study proposes to explore the relationship between psychological suzhi and the mental health indicators of positive and negative, and to investigate the influence of the factors on psychological suzhi to mental health, screen some factors which have greater influence on mental 
health, provide the basis for enhancing the pertinence of mental health education.

\section{Method}

\subsection{Subjects}

By using stratified sampling method, investigation was conducted among 734 Chinese college students, they were from 9 areas: Shandong Province, Hebei Province, Anhui Province, Zhejiang Province, Hunan Province, Sichuan Province, Guangdong Province, Shanghai municipality, and Chongqing municipality. This test was approved by the School Academic Ethic Committee, subjects signed the informed consent before the investigation, they can decide whether to participant in test regarding to their wishes. A total of 734 questionnaires were issued, some questionnaires which answered incompletely, answered all consistently, and answer was obviously regular were neglected, then according to the degree of lying (total score was $4-20$, the mean was $11.48 \pm 2.52$ ) and the degree of answer seriously (total score was 3 - 15, the mean was $12.28 \pm 2.24$ ), acquired 617 effective questionnaires, and effective rate was $84.0 \%$. It included 238 men (38.6\%), 372 women (60.3\%), and 7 subjects did not write. There were 227 freshmen (36.8\%), 200 sophomore students (32.4\%), and 187 junior students (30.3\%). Subjects between 16 and 24 years of age (mean $=19.62$, $\mathrm{SD}=1.30)$.

\subsection{Materials}

\subsubsection{College Student Psychological Suzhi Scale (CSPS)}

College Student Psychological Suzhi Scale (CSPS) was developed by Zhang Dajun team to measure psychological suzhi among college students [16]. After confirming the essential differences of psychological suzhi and psychological health [6], this scale was established successfully on the basic structure of the structure of psychological suzhi proposed by Zhang et al. for more than two years. Referring to the principle of mental quality, the writer collected topics according to usually and stably habitual behavior tendency and typical behavior characteristics. This scale can examine the picture of college students' psychological suzhi comprehensively from cognition to personality, diagnose underlying cause of college students' mental problem. It is an important tool to effectively assess the mental health of mental health education among college students. This scale consists of 118 items and adopts 5-point scoring method $(1=$ strongly disagree, $2=$ relatively disagree, $3=$ uncertainty, $4=$ relatively agree, 5 = strongly agree). It is composed of 3 subscales: (1) Cognition Quality, it consists of 44 items, includes 12 factors such as accuracy of reaction; (2) Personality Quality, it consists of 37 items, includes 10 factors such as knowledge seeking of motivation; (3) Adaptability, it consists of 30 items, includes 6 factors such as learning adaptability. Please refer to specific factors in Table 1. In present study, the Cronbach alphas of three subscales and total scale were $0.941,0.871,0.910$, and 0.960 , respectively.

\subsubsection{General Health Questionnaire-20 Item (GHQ-20)}

General Health Questionnaire (GHQ-20) was developed by Li Hong and Mei Jinrong to measure mental health in recent weeks [17]. This scale consists of 20 items, and it is composed of 3 subscales: (1) GHQ-self-affirmation, (2) GHQ-depression, and (3) GHQ-anxiety. The scale adopts yes-no scoring method ( $1=$ yes, $0=$ no). In present study, the Cronbach alphas of total scale were 0.755 , and the Cronbach alphas of three subscales were respectively $0.601,0.676$, and 0.694 .

\subsection{Data Analyses}

All data were managed and analysed by SPSS version 18.0. Statistical method includes descriptive statistics, correlation analysis, and regression analysis.

\section{Results}

\subsection{Correlation Analysis between Chinese College Students' Psychological Suzhi and Mental Health}

The score of psychological suzhi and three subscales are shown in Table 1: cognition quality, personality quality, adaptability existed positive correlation with the score of GHQ-self-affirmation $(p<0.001)$, and existed negative correlation with the sore of GHQ-20, GHQ-depression and GHQ-anxiety $(p<0.001)$. 
Table 1. Correlation analysis between Chinese college students’ psychological suzhi and mental health.

\begin{tabular}{|c|c|c|c|c|}
\hline & GHQ-20 & GHQ-self-affirmation & GHQ-depression & GHQ-anxiety \\
\hline Psychological suzhi & $-0.448^{* * *}$ & $0.439^{* * *}$ & $-0.262^{* * *}$ & $-0.259^{* * *}$ \\
\hline Cognition quality & $-0.357^{* * *}$ & $0.381^{* * *}$ & $-0.160^{* * *}$ & $-0.196 * *$ \\
\hline Accuracy of reaction & $-0.234^{* * *}$ & $0.242^{* * *}$ & $-0.113^{*}$ & $-0.134^{* * *}$ \\
\hline Agility of reaction & $-0.248^{* * *}$ & $0.321^{* * *}$ & -0.044 & $-0.113^{* *}$ \\
\hline Profundity of reaction & $-0.294^{* * *}$ & $0.348^{* * *}$ & -0.076 & $-0.157^{* * *}$ \\
\hline Curiosity of creativity & $-0.245^{* * *}$ & $0.261^{* * * *}$ & $-0.103^{*}$ & $-0.141^{* * *}$ \\
\hline Divergence of creativity & $-0.244^{* * *}$ & $0.305^{* * *}$ & -0.059 & $-0.113^{* *}$ \\
\hline Openness of creativity & $-0.284^{* * *}$ & $0.271^{* * *}$ & $-0.182^{* * * *}$ & $-0.156^{* * *}$ \\
\hline Teleonomy of practice & $-0.318^{* * *}$ & $0.315^{* * *}$ & $-0.194^{* * *}$ & $-0.165^{* * *}$ \\
\hline Contingency of practice & $-0.255^{* * *}$ & $0.301^{* * *}$ & $-0.088^{*}$ & $-0.120^{* *}$ \\
\hline Performance of practice & $-0.282^{* * *}$ & $0.302^{* * *}$ & $-0.130^{* *}$ & $-0.152^{* * *}$ \\
\hline Mobility of practice & $-0.263^{* * *}$ & $0.258^{* * *}$ & $-0.143^{* * *}$ & $-0.155^{* * *}$ \\
\hline Consciousness of metacognition & $-0.297^{* * *}$ & $0.270^{* * *}$ & $-0.180^{* * *}$ & $-0.194^{* * *}$ \\
\hline Monitoring of metacognition & $-0.227^{* * *}$ & $0.276^{* * * *}$ & -0.059 & $-0.113^{* *}$ \\
\hline Personality quality & $-0.473^{* * *}$ & $0.412^{* * *}$ & $-0.335^{* * *}$ & $-0.299^{* * *}$ \\
\hline Knowledge seeking of motivation & $-0.301^{* * *}$ & $0.316^{* * *}$ & $-0.163^{* * *}$ & $-0.148^{* * *}$ \\
\hline Achievement of motivation & $-0.256^{* * *}$ & $0.168^{* * *}$ & $-0.267^{* * *}$ & $-0.162^{* * *}$ \\
\hline Experience of emotion & $-0.370^{* * *}$ & $0.348^{* * * *}$ & $-0.218^{* * *}$ & $-0.229^{* * *}$ \\
\hline Control of emotion & $-0.222^{* * *}$ & $0.148^{* * *}$ & $-0.177^{* * *}$ & $-0.187^{* * *}$ \\
\hline Hardiness of volition & $-0.174^{* * *}$ & $0.243^{* * *}$ & -0.052 & -0.032 \\
\hline Possessiveness of volition & $-0.242^{* * *}$ & $0.208^{* * *}$ & $-0.177^{* * *}$ & $-0.149^{* * *}$ \\
\hline Independence of volition & $-0.204^{* * *}$ & $0.157^{* * *}$ & $-0.145^{* * *}$ & $-0.158^{* * *}$ \\
\hline Cognition of self & $-0.334^{* * *}$ & $0.253^{* * *}$ & $-0.248^{* * *}$ & $-0.254^{* * *}$ \\
\hline Experience of self & $-0.400^{* * *}$ & $0.294^{* * *}$ & $-0.331^{* * *}$ & $-0.290^{* * *}$ \\
\hline Control of self & $-0.216^{* * *}$ & $0.258^{* * *}$ & $-0.087^{*}$ & $-0.085^{*}$ \\
\hline Adaptability & $-0.376^{* * *}$ & $0.392^{* * *}$ & $-0.199^{* * *}$ & $-0.194^{* * *}$ \\
\hline Learning adaptability & $-0.269^{* * *}$ & $0.300^{* * *}$ & $-0.127^{* *}$ & $-0.126^{* *}$ \\
\hline Living adaptability & $-0.326^{* * *}$ & $0.312^{* * *}$ & $-0.223^{* * *}$ & $-0.161^{* * *}$ \\
\hline Physiological adaptability & $-0.281^{* * *}$ & $0.258^{* * *}$ & $-0.145^{* * *}$ & $-0.200^{* * *}$ \\
\hline Social adaptability & $-0.344^{* * * *}$ & $0.392^{* * * *}$ & $-0.123^{* *}$ & $-0.181^{* * *}$ \\
\hline Interpersonal adaptability & $-0.288^{* * *}$ & $0.304^{* * *}$ & $-0.146^{* * *}$ & $-0.149^{* *}$ \\
\hline Vocational adaptability & $-0.259^{* * *}$ & $0.318^{* * *}$ & $-0.106^{* *}$ & $-0.090^{*}$ \\
\hline
\end{tabular}

Note: ${ }^{*} p<0.05, \stackrel{* *}{p} p<0.01, \stackrel{* * *}{p} p<0.001$. 


\subsection{Linear Regression Analysis between Psychological Suzhi and Mental Health among Chinese College Students}

\subsubsection{Linear Regression Analysis between the Total Score of Psychological Suzhi and Mental Health among Chinese College Students}

In the linear regression analysis, GHQ-20, GHQ-self-affirmation, GHQ-depression and GHQ-anxiety are used as the dependent variable, and the score of psychological suzhi are defined as the independent variable (see Table 2). Psychological suzhi was significant predictor for GHQ-20, GHQ-self-affirmation, GHQ-depression, and GHQ-anxiety $(\beta=-0.448,0.439,-0.262,-0.259, p<0.001)$. The variance of Chinese college students' psychological suzhi explained were $19.9 \%, 19.1 \%, 6.7 \%$, and $6.5 \%$, respectively.

\subsubsection{Linear Regression Analysis between Cognition Quality of Psychological Suzhi and Mental Health among Chinese College Students}

The result of the linear regression analysis indicated that the score of cognition quality of psychological suzhi was significant predictor for GHQ-20, GHQ-self-affirmation, GHQ-depression, and GHQ-anxiety $(\beta=-0.357$, $0.381,-0.160,-0.196, p<0.001)$. Cognition quality accounted for the variance was respectively $12.6 \%, 14.3 \%$, $2.4 \%$, and $3.7 \%$.

Upon further multivariate linear regression analysis, the result showed that 7 factors of cognition quality went into the equation of GHQ-20 and three subscales. A table is shown below regarding to the results (see Table 3).

Table 2. Linear regression analysis between the total score of psychological suzhi and mental health among Chinese college students.

\begin{tabular}{cccc}
\hline Dependent variable & $\beta$ & adj $R^{2}$ & $F$ \\
\hline GHQ-20 & $-0.448^{* * *}$ & 0.199 & $133.551^{* * *}$ \\
GHQ-self-affirmation & $0.439^{* * *}$ & 0.191 & $127.041^{* * *}$ \\
GHQ-depression & $-0.262^{* * *}$ & 0.067 & $39.321^{* * *}$ \\
GHQ-anxiety & $-0.259^{* * *}$ & 0.065 & $38.339^{* * *}$ \\
\hline
\end{tabular}

Note: ${ }^{*} p<0.05,{ }^{* *} p<0.01,{ }^{* * *} p<0.001$.

Table 3. Linear regression analysis between cognition quality of psychological suzhi and mental health among Chinese college students.

\begin{tabular}{|c|c|c|c|c|}
\hline Dependent variable & Independent variable & $\beta$ & $\operatorname{adj} R^{2}$ & $F$ \\
\hline \multirow{4}{*}{ GHQ-20 } & Teleonomy of practice & $-0.152^{* *}$ & \multirow{3}{*}{0.124} & \multirow{3}{*}{$27.700^{* * *}$} \\
\hline & Consciousness of metacognition & $-0.141^{* *}$ & & \\
\hline & Profundity of reaction & $-0.131^{*}$ & & \\
\hline & Profundity of reaction & $0.145^{*}$ & \multirow{4}{*}{0.151} & \multirow{4}{*}{$26.191^{* * *}$} \\
\hline \multirow{4}{*}{ GHQ-self-affirmation } & Contingency of practice & $0.107^{*}$ & & \\
\hline & Agility of reaction & $0.132^{*}$ & & \\
\hline & Teleonomy of practice & $0.104^{*}$ & & \\
\hline & Teleonomy of practice & $-0.122^{*}$ & \multirow{4}{*}{0.060} & \multirow{4}{*}{$10.031^{* * *}$} \\
\hline \multirow{3}{*}{ GHQ-depression } & Consciousness of metacognition & $-0.172^{* *}$ & & \\
\hline & Monitoring of metacognition & $0.174^{* *}$ & & \\
\hline & Openness of creativity & $-0.111^{*}$ & & \\
\hline GHQ-anxiety & Consciousness of metacognition & $-0.199^{* * *}$ & 0.038 & $23.293^{* * *}$ \\
\hline
\end{tabular}

Note: ${ }^{*} p<0.05,{ }^{* *} p<0.01,{ }^{* * *} p<0.001$. 


\subsubsection{Linear Regression Analysis between Personality Quality of Psychological Suzhi and Mental Health among Chinese College Students}

The result of the linear regression analysis showed that the score of personality quality of psychological suzhi was significant predictor for GHQ-20, GHQ-self-affirmation, GHQ-depression, and GHQ-anxiety $(\beta=-0.473$, $0.412,-0.335,-0.299, p<0.001)$. Personality quality accounted for the variance was respectively $22.2 \%, 16.8 \%$, $11.0 \%$, and $8.8 \%$.

Upon further multivariate linear regression analysis, the result showed that 8 factors of personality quality went into the equation of GHQ-20 and three subscales. A table is shown below regarding to the results (see Table 4).

\subsubsection{Linear Regression Analysis between Adaptability of Psychological Suzhi and Mental Health among Chinese College Students}

The result of linear regression analysis showed that the score of adaptability of psychological suzhi was significant predictor for GHQ-20, GHQ-self-affirmation, GHQ-depression, and GHQ-anxiety ( $\beta=-0.376,0.392$, $-0.199,-0.194, p<0.001)$. Personality quality accounted for the variance was respectively $14.0 \%, 15.2 \%$, $3.80 \%$, and $3.60 \%$.

Upon further multivariate linear regression analysis, the result showed that 3 factors of adaptability went into the equation of GHQ-20 and three subscales. A table is shown below regarding to the results (see Table 5).

\section{Discussion}

The results of correlation analysis displayed that there was a significant correlation between Chinese college students' psychological suzhi and mental health, which was similar to the existing research [8] [10] [11]. Likewise, the result also indicated that the correlation between psychological suzhi and its three dimensions with GHQ-self-affirmation was higher than the correlation between psychological suzhi and its three dimensions with GHQ-depression and GHQ-anxiety. With few exceptions, the factors of three psychological dimensions presented

Table 4. Linear regression analysis between personality quality of psychological suzhi and mental health among Chinese college students.

\begin{tabular}{|c|c|c|c|c|}
\hline Dependent variable & Independent variable & $\beta$ & $\operatorname{adj} R^{2}$ & $F$ \\
\hline \multirow{4}{*}{ GHQ-20 } & Experience of self & $-0.230^{* * *}$ & \multirow{4}{*}{0.238} & \multirow{4}{*}{$47.117^{* * *}$} \\
\hline & Experience of emotion & $-0.222^{* * *}$ & & \\
\hline & Possessiveness of volition & $-0.136^{* *}$ & & \\
\hline & Knowledge seeking of motivation & $-0.110^{*}$ & & \\
\hline \multirow{6}{*}{ GHQ-self-affirmation } & Experience of emotion & $0.243^{* * *}$ & \multirow{6}{*}{0.197} & \multirow{6}{*}{$25.144^{* * *}$} \\
\hline & Possessiveness of volition & $0.130^{* *}$ & & \\
\hline & Knowledge seeking of motivation & $0.152^{* *}$ & & \\
\hline & Experience of self & $0.134^{* *}$ & & \\
\hline & Achievement of motivation & $-0.110^{*}$ & & \\
\hline & Hardiness of volition & $0.094^{*}$ & & \\
\hline \multirow{2}{*}{ GHQ-depression } & Experience of self & $-0.275^{* * *}$ & \multirow{2}{*}{0.140} & \multirow{2}{*}{$49.043^{* *}$} \\
\hline & Achievement of motivation & $-0.192^{* * *}$ & & \\
\hline \multirow{3}{*}{ GHQ-anxiety } & Experience of self & $-0.168^{* *}$ & \multirow{3}{*}{0.107} & \multirow{3}{*}{$24.469^{* * *}$} \\
\hline & Experience of emotion & $-0.138^{* *}$ & & \\
\hline & Cognition of self & $-0.117^{*}$ & & \\
\hline
\end{tabular}

Note: ${ }^{*} p<0.05,{ }^{* *} p<0.01,{ }^{* * *} p<0.001$. 
Table 5. Linear regression analysis between adaptability of psychological suzhi and mental health among Chinese college students.

\begin{tabular}{|c|c|c|c|c|}
\hline Dependent variable & Independent variable & $\beta$ & $\operatorname{adj} R^{2}$ & $F$ \\
\hline \multirow{3}{*}{ GHQ-20 } & Social adaptability & $-0.215^{* * *}$ & \multirow{3}{*}{0.156} & \multirow{3}{*}{$37.636^{* * * *}$} \\
\hline & Living adaptability & $-0.159^{* *}$ & & \\
\hline & Physiological adaptability & $-0.119^{* *}$ & & \\
\hline \multirow{2}{*}{ GHQ-self-affirmation } & Social adaptability & $0.315^{* * *}$ & \multirow{2}{*}{0.164} & \multirow{2}{*}{$58.995^{* * *}$} \\
\hline & Living adaptability & $0.144^{* *}$ & & \\
\hline GHQ-depression & Living adaptability & $-0.231^{* * *}$ & 0.052 & $33.393^{* * * *}$ \\
\hline \multirow{2}{*}{ GHQ-anxiety } & Physiological adaptability & $-0.154^{* *}$ & \multirow{2}{*}{0.050} & \multirow{2}{*}{$16.703^{* * * *}$} \\
\hline & Social adaptability & $-0.116^{*}$ & & \\
\hline
\end{tabular}

Note: ${ }^{*} p<0.05,{ }^{* *} p<0.01,{ }^{* * *} p<0.001$.

the same rule. According to the theory of complete mental health [13]-[15], GHQ-self-affirmation can be considered positive indicator of mental health, GHQ-depression and GHQ-anxiety can be considered negative indicator of mental health. Taken together, the result was observed that the relationship between psychological suzhi and positive mental health condition is closer, and developmental function of psychological suzhi may be higher than therapeutic function. Existing research usually used the subject well-being and the meaning in life as the metric of positive mental health [18] [19], this research only used GHQ-self-affirmation to measure positive mental health. Therefore, this conclusion needs to confirm from further study.

The results of regression analysis indicated that psychological suzhi and its three dimensions were significant predictors for GHQ-20 and its three subscales. This reveals that College students' psychological suzhi has powerful impact on both positive and negative indicator of mental health. The results complement empirical study about the relationship of psychological suzhi and mental health [10] [11] [20], and support the educational concept which improve mental health condition among College students by improving psychological suzhi [21]. Meanwhile, the results indicated that the variance of GHQ-self-affirmation explained by psychological suzhi and its three dimensions among Chinese college students is higher, we can observe that the relationship between psychological suzhi and positive mental health condition is closer. This point is identical to correlation analysis, and does not be repeated here.

The results of regression analysis also indicated that, in the three dimensions of psychological suzhi, personality quality had the most impact on mental health and its three subscales, it accounted for the total variance was $22.2 \%$. Personality quality is dynamic ingredient of psychological suzhi, it does not participate in concrete operations directly, but it has dynamic effect and adjustment function to cognitive performance [4] [5] [22] [23]. The results show that personality quality, as dynamic ingredient, is greater predictor for mental health than cognition quality and adaptability. This point gets the same result with the study among middle school students [10]. This enlighten that we can give priority to train personality quality for college students.

Another outcome of this study is to obtain psychological suzhi factors which are significant predictors for GHQ-20, GHQ-self-affirmation, GHQ-depression, and GHQ-anxiety by adopting multiple linear regression analysis method. There are 12 psychological suzhi factors are significant predictors for GHQ-self-affirmation which was viewed as positive indicator of mental health. The 12 factors can positively predict GHQ-self-affirmation except achievement of motivation. Achievement of motivation refers to the desire of succeed as the impetus to move forward, belongs to the motivation which shows goal orientation. This means that you will reduce the degree of self-affirmation, and neglect experience of successes and failures, if you do not obtain achievement which you expect. There are 11 psychological suzhi factors are significant predictors for GHQ-depression and GHQ-anxiety, all of them are positive predictors for GHQ-depression and GHQ-anxiety which were regarded as negative indicator of mental health. The reason could be found as the following. The higher strength to monitor the own method and so on, the easier way to discover own shortcomings, hence it could cause the depression degree due to the strong dissatisfaction of self. In this case the resting-sate which is a part of depression may have the similar component with this state. In the screened factors, teleonomy of practice, experience 
of emotion, experience of self, achievement of motivation, social adaptability and living adaptability were significant predictors for positive and negative indicator of mental health at the same time. It is believed that these 6 factors are core indicators to predict complete mental health condition, can be considered first in college students' mental health education.

This study confirmed that psychological suzhi plays a role in mental health, and obtained psychological suzhi factors which were significant predictors. This point provides a good way to carry on specific mental health education. But this study also has its limitation. Current study only adopted cross-sectional survey method, if we want to provide valid proof which is based on the time for the relationship and predicted effect of psychological suzhi and mental health, we need to conduct further longitudinal survey.

\section{Conclusions}

1) There exists a significant correlation between Chinese college students' psychological suzhi and mental health, meaning that a significant correlation between psychological suzhi and complete mental health exists. Relative to negative mental health, the relationship between psychological suzhi and positive mental health is closer.

2) The three dimensions of college students' psychological suzhi are significant predictors for mental health condition. Relative to cognition quality and adaptability, personality quality is a greater predictor for mental health condition.

3) There are 23 factors of psychological suzhi that are significant predictors for negative and positive indicator of mental health. In these factors, teleonomy of practice, experience of emotion, experience of self, achievement of motivation, social adaptability, and living adaptability all have effect for negative and positive mental health, and are core factors to influence mental health.

\section{Acknowledgements}

This study was supported by the Science foundation for Young Scholars of Jiangxi Province (20151BAB215033), the postdoctoral Science Foundation of Jiangxi Province (2014KY52, 2014RC11), and Dr. Start-up fund project of Jiangxi normal university (the concise psychological suzhi scale establishment and its mechanism of action research on mental health).

\section{References}

[1] Li, Y.A. and Wei, Y.L. (2007) The Psychometric Properties of GHQ-20 in University Student. China Journal of Health Psychology, 15, 11-13.

[2] Wang, X.Q. and Tan, L. (2014) The Research of Psychological Suzhi among Sichuan, Chongqing and Hubei Middle School Students. Educational Research and Experiment, No. 1, 86-91.

[3] Xin, Z.Q., Zhang, M. and He, L. (2012) Changes in College Students’ Mental Health: A Cross-Temporal Meta-Analysis. Acta Psychologica Sinica, 44, 664-679. http://dx.doi.org/10.3724/SP.J.1041.2012.00664

[4] Zhang, D.J., Feng, Z.Z., Guo, C. and Chen, X. (2000) Problems on Research of Students' Mental Quality. Journal of Southwest China Normal University (Humanities and Social Sciences Edition), 26, 56-62.

[5] Zhang, D.J. (2003) On Man’s Mental Quality. Studies of Psychology and Behavior, 2, 143-146.

[6] Zhang, D.J. and Wang, X.Q. (2012) An Analysis of the Relationship between Mental Health and Psychological Suzhi: From Perspective of Connotation and Structure. Journal of Southwest University (Social Sciences Edition), 38, 69-74.

[7] Zhang. H.Z. (2007) Mental Hygiene and Psychological Education: Concept, Evaluation, Criteria. Journal of Gannan Normal University, 28, 6-10.

[8] Wang, X.Q. and Zhang. D.J. (2012) Looking beyond PTH and DFM: The Relationship Model between Psychological Suzhi and Mental Health. Journal of Southwest University (Social Sciences Edition), 38, 67-74.

[9] Zhang, D.J. (2012) Integrating Adolescents' Mental Health and Psychological Suzhi Cultivation. Journal of Psychological Science, 3, 530-536.

[10] Wang, X.Q. and Zhang, D.J. (2015) The Relationship Model of Psychological Suzhi and Mental Health among Adolescents. China: Science Press, Beijing, 102-268.

[11] Xie, J., Yu, H.Y., Feng, Z.Z., Yang, G.Y. and Jiang, J. (2011) Correlation between Mental Quality and Personality and Mental Health of Young Army Men. Progress in Modern Biomedicine, 11, 2163-2167. 
[12] Xin, Z.Q., Zhang, M. and He, L. (2012) Changes in College Students’ Mental Health: A Cross-Temporal Meta-Analysis. Acta Psychologica Sinica, 44, 664-679.

[13] Boey, K.W. and Chiu, H.F.K. (1998) Assessing Psychological Well-Being of the Old-Old: A Comparative Study of GDS-15 and GHQ-12. Clinical Gerontologist, 19, 65-75.

[14] Doll, B. (2008) The Dual-Factor Model of Mental Health in Youth. School Psychology Review, 37, 69-73.

[15] Robitschek, C. and Keyes, C.L.M. (2009) Keyes’s Model of Mental Health with Personal Growth Initiative as a Parsimonious Predictor. Journal of Counseling Psychology, 56, 321-329. http://dx.doi.org/10.1037/a0013954

[16] Gong, L. (2011) Amendment of College Students’ Psychological Suzhi Scale. M.S. Thesis, Southwest University, Chongqing.

[17] Li, H. and Mei, J.R. (2002) Assessing Psychological Well-Being of College Student: Psychometric Properties of GHQ-20. Psychological Development and Education, 18, 75-79.

[18] Keyes, C.L.M. (2006) Mental Health in Adolescence: Is America’s Youth Flourishing? American Journal of Orthopsychiatry, 76, 395-402. http://dx.doi.org/10.1037/0002-9432.76.3.395

[19] Keyes, C.L.M. (2007) Promoting and Protecting Mental Health as Flourishing a Complementary Strategy for Improving National Mental Health. American Psychologist, 62, 95-108. http://dx.doi.org/10.1037/0003-066X.62.2.95

[20] Wang, X.Q. and Zhang, D.J. (2012) The Criticism and Amendment for the Dual-Factor Model of Mental Health: From Chinese Psychological Suzhi Research Perspectives. International Journal of Clinical Medicine, 5, 319-327. http://dx.doi.org/10.4236/ijcm.2012.35063

[21] Zhang, D.J. (2006) Study on the Theories of Mental Health Education in the University. Journal of Southwest China Normal University (Humanities and Social Sciences Edition), 32, 130-136.

[22] Zhang, D.J. (2010) Psychological Suzhi and Its Structure. In: Columbus, A.M., Ed., Advances in Psychology Research, Nova Publication, New York, 239-250.

[23] Zhang, D.J., Wang, J.L. and Yu, L. (2011) Methods and Implementary Strategies on Cultivating Students' Psychological Suzhi. Nova Science Publishers, New York. 